This is an Accepted Manuscript of an article published by IOS Press in "Lournal of Alzheimer's Disease", available online: http://content.iospress.com/articles/journal-of-alzheimersdisease/jad160149

\title{
Blockade of the Interaction of Calcineurin with FOXO in Astrocytes Protects Against Amyloid- $\beta$ - Induced Neuronal Death
}

Ana M. Fernandeza, ${ }^{\mathrm{b}}$, Ruben Hervasa, Manuel Dominguez-Frailea, ${ }^{\mathrm{b}}$, Victoria Navarro Garridob, ${ }^{\mathrm{c}}$, Patricia Gomez-Gutierrez ${ }^{\mathrm{d}}$, Miguel Vega ${ }^{\mathrm{e}}$, Javier Vitoricab, ${ }^{\mathrm{c}}, \mathrm{Juan}_{\mathrm{N}} \mathrm{J}$. Perez ${ }^{\mathrm{d}}$ and Ignacio Torres Alemana,b,*

${ }^{a}$ Cajal Institute, CSIC, Madrid, Spain

${ }^{\mathrm{b}}$ CIBERNED, Madrid, Spain

${ }^{c}$ IBIS, CSIC-Universidad de Sevilla, Spain

${ }^{\mathrm{d}}$ GBMI, Universitat Politecnica de Catalunya, Barcelona, Spain

${ }^{\mathrm{e}}$ Allinky Biopharma, Madrid, Spain 
*Correspondence to: Ignacio Torres Aleman, Cajal Institute,

Avda Dr Arce 37, 28002 Madrid, Spain. Tel.: +34 915854723;

Fax: +34 915854754; E-mail: torres@cajal.csic.es.

Keywords: Alzheimer's disease, astrocytes, calcineurin, decoy compounds, FOXO 


\begin{abstract}
Astrocytes actively participate in neuro-inflammatory processes associated to Alzheimer's disease (AD), and other brain pathologies. We recently showed that an astrocyte-specific intracellular signaling pathway involving an interaction of the phosphatase calcineurin with the transcription factor $\mathrm{FOXO} 3$ is a major driver in $\mathrm{AD}$-associated pathological inflammation, suggesting a potential new druggable target for this devastating disease. We have now developed decoy molecules to interfere with calcineurin/FOXO3 interactions, and tested them in astrocytes and neuronal co-cultures exposed to amyloid- $\beta$ (A $\beta$ ) toxicity. We observed that interference of calcineurin/FOXO3 interactions exerts a protective action against A-induced neuronal death and favors the production of a set of growth factors that we hypothesize form part of a cytoprotective pathway to resolve inflammation. Furthermore, interference of the A-induced interaction of calcineurin with $\mathrm{FOXO} 3$ by decoy compounds significantly decreased amyloid- $\beta$ protein precursor (A $\beta P P)$ synthesis, reduced the A $\beta P P$ amyloidogenic pathway, resulting in lower Alevels, and blocked the expression of pro-inflammatory cytokines TNFa and IL-6 in astrocytes. Collectively, these data indicate that interrupting pro-inflammatory calcineurin/FOXO3 interactions in astrocytes triggered by $A \beta$ accumulation in brain may constitute an effective new therapeutic approach in AD. Future studies with intranasal delivery, or brain barrier permeable decoy compounds, are warranted.
\end{abstract}




\section{INTRODUCTION}

An intense search for new druggable targets to treat Alzheimer's disease (AD) is underway, as no effective treatment is yet in sight. While analyzing the insulin-like growth factor I (IGF-I) pathway as a paradigm of endogenous neuroprotective signalling underlying responses to brain disease, we recently unveiled an astrocyte-specific pathway involved in resolution of inflammation triggered by diverse types of brain injuries, including $\mathrm{AD}[1,2]$. This pathway recruited the phosphatase calcineurin and the transcription factor FOXO, both already proposed to be involved in AD pathology [3, 4]. Specifically, we observed that IGF-I disrupts the association of calcineurin with FOXO3 that takes place in response to pro-inflammatory stimuli such as TNFa or amyloid- $\beta$ (A $\beta)$. Indeed, calcineurin/FOXO3 complexes were found in the brain of $\mathrm{AD}$ patients, but not in age-matched non-demented controls [2]. In this way, IGF-I promotes resolution of the neuro-inflammatory cascade by halting downstream activation of the canonical pro-inflammatory transcription factor NFKB [2]. We hypothesized that inhibition of the association of calcineurin with FOXO3 may constitute a feasible target to prevent neuro-inflammation associated to $\mathrm{AD}$ and other diseases, and in this way halt or delay neuronal demise.

Using computer-assisted drug design, we discovered a series of decoy compounds that interfere with the association of calcineurin with $\mathrm{FOXO} 3$ in response to pro-inflammatory stimuli. Two of these peptide-mimetics showed good neuroprotective profile in an in vitro system mimicking A-induced neuronal injury.

\section{MATERIALS AND METHODS}

Materials and animals

C57BL6/J (wild type, wt) and APP/PS1 with C57BL6/J background (a kind gift from P. Mouton, NIH) mice were used. Animals were used according to European (86/609/EEC \& 2003/65/EC, European Council Directives) guidelines and studies were approved by the Madrid Government Bioethics Committee.

Bacterial lypopolysaccharide (LPS) was from Sigma (St. Louis, USA), and A $\beta 40$ was from Polypeptide (Strasbourg, France). Antibodies against activated caspase 3 (Cell Signaling, USA, a marker of apoptotic cells), $\mathrm{A} \beta$ (6E10 from BioLegend (1/6000), and 82E1 from IBL (1/5000) were used in combination), $\beta 3$-tubulin (1/5000; Promega, USA, a neuronal marker), GFAP (Sigma, an astrocyte marker), $\beta$-tubulin (1/2000; Sigma), calcineurin (Cell Signaling), FOXO3 (Cell Signaling), IGF-I (Abcam, UK), IGF-II (Abcam), FGF-8 (Santa Cruz, USA), sA $\beta P$ Pa (IBL-America, USA), and BSA (Santa Cruz) were all used at a 1/1000 dilution except when indicated otherwise. Secondary antibodies were goat anti- 
rabbit or mouse HRP- (1/20000, BioRad, USA) or Alexa Fluor 488 or 594 donkey antimouse or rabbit (1/1000, Molecular Probes, USA).

Protein expression and purification

Vectors pGEX-6P-1-human Calcineurin and pGEX-4T3-human FOXO3 were purchased from Addgene (plasmids \#13251 and \#1790). Recombinant calcineurin and FOXO3 GSTfusion proteins were expressed in E. coli BL21(DE3) (Invitrogen) strain [5]. Cultures were grown at $280 \mathrm{rpm}$ and $37{ }^{\circ} \mathrm{C}$ until they reached an OD595 of $0.5-0.8$, and expression was induced by addition of $1 \mathrm{mM}$ IPTG for $4 \mathrm{~h}$. Bacteria cells were then lysed with $1 \mathrm{mg} / \mathrm{ml}$ lysozyme, $1 \%$ Triton X-100, as reported previously [6] with modifications, such as the addition of $0.5 \%$ Tween- 20 and sonication pulses. Recombinant proteins were purified by GST-affinity chromatography using GSTrap HP columns (GE Healthcare, USA) on an FPLC apparatus ( ÄKTA Purifier, GE Healthcare), with a buffer system consisting of 10 $\mathrm{mM}$ sodium phosphate, $0.14 \mathrm{M} \mathrm{NaCl}, \mathrm{pH} 7.4$ and $50 \mathrm{mM}$ Tris- $\mathrm{HCl}, 10 \mathrm{mM}$ reduced glutathione, $\mathrm{pH} 8.0$ for binding and elution, respectively. A size-exclusion chromatography on a HiLoad 16/60 200 PG column (GE Healthcare) using PBS pH 7.4 was performed after GST-affinity chromatography purification. For both proteins, $\sim 90 \%$ purity at the end of the purification procedures was reached, as estimated by SDS-PAGE electrophoresis. Finally, the purified fractions were concentrated (up to $50 \mathrm{M}$ ) and the buffer exchanged to $1 \mathrm{mM}$ pH 7.4 by ultrafiltration using Amicon Ultra-15 10K filters (Millipore, Germany), prior to performing the pull-down assays. Protein concentration was determined by absorbance at $280 \mathrm{~nm}$ using its calculated molar extinction coefficient.

Pull-down assays

GST fusioned to wild type calcineurin or wild type FOXO3 proteins were employed as baits in pull-down experiments. Glutathione Sepharose 4B prepack columns (GE Healthcare) were washed with binding buffer (PBS pH 7.4), and equilibrated with PBS, 1\% Triton X-100 for $30 \mathrm{~min}$ at room temperature. One $\mathrm{ml}$ of lysates of $\mathrm{CHO} \mathrm{K} 1$ cells transfected with calcineurin (a kind gift of JM Redondo, CNIC) or FOXO3 (Addgene) mutant constructs was added to the GST fusion protein columns for $30 \mathrm{~min}$. Columns were then washed five times with binding buffer, and the bound protein was eluted in $50 \mathrm{mM}$ Tris- $\mathrm{HCl}, 10 \mathrm{mM}$ reduced glutathione $\mathrm{pH}$ 8.0, dissolved in 5\% $\beta$-mercaptoethanol Laemli buffer and analyzed by western blotting.

Virtual screening

Our strategy for the discovery of new hits was carried out by virtual screening, using as template a pharmacophore defined from the analysis of the crystal structure of the complex 
of calcineurin with the calcineurin-inhibiting domain of the African Swine Fever Virus Protein A238L (entry 4F0Z of the pdb)[7]. In this structure, the A238L peptide competitively inhibits calcineurin by occupying the critical substrate recognition site $\varphi \mathrm{LxVP}$, while leaving the catalytic center fully accessible (Fig. 1A).

Analysis of the crystal structure allowed identifying several chemical groups putatively critical for the peptide-calcineurin interaction. Accordingly, we defined a five point pharmacophore that consisted in two hydrogen bond acceptors points (F1 and F2), one hydrogen bond donor (F3), and two hydrophobic or aromatic points (F4 and F5). An exclusion volume was added to simulate the receptor surface, in order to avoid molecules clashing with the receptor. The pharmacophore was used as query for a search on the leadlike compound database of the MOE program (ca. 650.000 compounds) [8]. The search yielded a total of 3285 hits that fulfilled the pharmacophore hypothesis. These molecules were subsequently submitted to a docking process onto the $\varphi \mathrm{LxVP}$ site, using the program Glide with the SP scoring function [9]. Molecules in their bioactive conformations were subsequently analyzed for pharmacophore fulfillment, revealing that only 509 molecules fulfilled at least four pharmacophoric points. Of this subset, only those with a docking score (SP) greater than 5 were subsequently grouped into 20 clusters, following a hierarchical clustering analysis. For this purpose, molecules were classified by means of a three-point pharmacophore fingerprint, and their distance measured by the Tanimoto coefficient, using the program Canvas of Schrödinger software [10]. From the cluster analysis we selected one representative of each cluster for testing. However, only 11 molecules could be acquired and tested (compounds A CN 001, A CN 002, A CN 003, A CN 005, A CN 006, A CN 008, A CN 009, A CN 010, A CN 011, A CN 013, and A CN 014). Based on the results, another 10 compounds were selected from the clusters where active compounds had been found (compounds A CN 005 and A CN 006): A CN 15 to A $\mathrm{CN} 24$. Finally, we selected 6 additional compounds structurally related to the most active compound found (A CN 006): A CN 027 to A CN 32.

In vitro cultures

Pure astroglial cultures ( $>95 \%$ GFAP+, OX-42-, A2B5- cells) were prepared as described [1]. Briefly, postnatal (day 3-4) mice were rapidly decapitated to minimize suffering, and their brains dissected and immersed in ice-cold DMEM/F-12 (Invitrogen) supplemented with $10 \%$ fetal bovine serum (FBS). Cortex and hippocampus were removed and cut into 1 $\mathrm{mm}$ pieces. Tissue fragments were dissociated mechanically, and the resulting cell suspension was centrifuged (1000 rpm/10 min) and plated in DMEM/F-12 with 10\% FBS, $5 \times 10^{5}$ cells per well. Cultures were allowed to grow for 2 days. Pure neuronal cultures 
were obtained as described [1]. Astrocyte-neuron co-cultures were prepared as follows: Astrocytes were plated, and when they were $70 \%$ confluent, the medium was removed and neurons were plated onto them with freshly added medium (Neurobasal+B27 (Invitrogen), $4 \mathrm{mM}$ glutamine, and $25 \mathrm{mM} \mathrm{KCl}$ ). Cultures were allowed to grow for 7 days. In experiments using wild type (wt) cultures, cells were exposed for $16 \mathrm{~h}$ to $10 \mathrm{nM}$ LPS or 2 $\mu \mathrm{M} \mathrm{A} \beta$ prior to adding decoy compounds, and cell death was assessed $2 \mathrm{~h}$ later. Cytoxicity was determined with the MTT assay following the manufacturer's instructions.

Immunofluorescence

Immunocytochemistry was performed as described [11]. Cells were plated on $20 \mathrm{~mm}$ coverslips and fixed. Coverslips were blocked with 5\% normal horse serum, and incubated overnight at $4{ }^{\circ} \mathrm{C}$ with the respective primary antibody in phosphate buffer $(\mathrm{PB})$ containing $0.1 \%$ bovine albumin, $3 \%$ horse serum, and $0.2 \%$ Triton $\mathrm{X}-100$. After several washes in PB, sections were incubated with an Alexa Fluor 488 or 594 donkey anti-mouse or rabbit (1/1000, Molecular Probes). Omission of primary antibody was used as control. Neuronal death was determined by a single investigator in blinded cultures, as described [12]. Confocal analysis was performed in a Leica microscope (Leica Microsystems, Germany).

Immunoassays

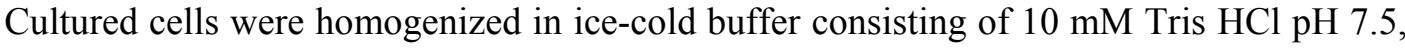
$150 \mathrm{mM} \mathrm{NaCl}, 1 \mathrm{mM}$ EDTA, $1 \mathrm{mM}$ EGTA, 1\% Triton X-100, 0.5\% NP40, $1 \mathrm{mM}$ sodium orthovanadate, and a protease inhibitor cocktail (Sigma) plus $2 \mathrm{mM}$ PMSF. Western blot and immunoprecipitation were per-formed as described [1]. Western blot membranes were reblotted with either the same antibody used for immunoprecipitation, or with anti- $\beta$ tubulin or anti-bovine serum albumin as internal standards, and to normalize for protein load. Levels of the protein under study were expressed relative to protein load in each lane. Densitometric analysis was performed using Quantity One software (BioRad).

Quantitative PCR

The first strand cDNA was synthesized from RNA using the RT $^{2}$ First Strand Kit (SABiosciences). The $\Delta \Delta \mathrm{Ct}$ method was used to analyze the expression level of each gene using the Data Analysis Template provided by SABiosciences. After PCR, the dissociation curve for each gene was examined to exclude those with nonspecific amplification, or with undetectable expression. Quantitative real-time PCR was performed using an ABI Prism 7500 detection System (Applied Biosystems).

Statistics 
Statistical analyses were performed with a t-test when comparing two groups, and a twoway ANOVA for comparing multiple groups. Statistical significance was established when $\mathrm{p}<0.05$. Results shown are mean $\pm \mathrm{SEM}$.

\section{RESULTS}

Using pull-down assays with several FOXO3 and calcineurin mutants, we mapped regions involved in FOXO3-calcineurin interaction at residues 468-673 of FOXO3 and 347-521 of calcineurin (Supplementary Figure 1A). We then used computer-assisted design to discover a series of decoy compounds directed to interfere with the binding region of calcineurin with FOXO3. Different series of such compounds (ACN01-ACN032) were tested in cultured astrocytes under inflammatory challenge, using LPS $(10 \mathrm{nM})$ to promote association of calcineurin with FOXO3 [1]. Using this assay, we identified compounds ACN018 and ACN030, -two ((1,3,4-oxadiazol-2-yl) methyl) carbamate analogs with diverse substituents, displaying the greatest inhibition on calcineurin/ FOXO3 interactions (Fig. 1B), without affecting cell viability (Supplementary Figure 1B).

Next, we tested the capacity of these compounds to interfere with A-induced neuronal death. We hypothesized that if they are able to counteract pro-inflammatory signaling in astrocytes and re-direct them toward resolution of inflammation, treated astrocytes would become more neuroprotective. Importantly, under culture conditions only neurons die upon $\mathrm{A} \beta$ challenge, as indicated by neuronal-specific immunostaining of activated caspase 3 , a marker of apoptosis (Fig. 1C). On the other hand, neurons exposed to A $\beta$ do not show calcineurin/FOXO complexing (Fig. 1D). The latter indicates that calcineurin/FOXO3 complexes in co-cultures exposed to pro-inflammatory challenge originate only in astrocytes, confirming previous observations of an essential role of astrocytes in mediating A $\beta$ neurotoxicity in culture [13]. When either compound 018 or 030 were added to cocultures exposed to $\mathrm{A} \beta(2 \mu \mathrm{M})$, levels of activated caspase 3 drastically decreased, as compared to control co-cultures (Fig. 1E). Furthermore, protection against A $\beta$-induced neuronal death provided by these compounds was linked to a decrease in the reported $A \beta$ induced increase in A $\beta P P$ [14], and in its resultant increased amyloidogenic processing. Thus, as shown in Fig. 2, A $\beta P P$ mRNA and $A \beta$ levels were reduced after addition of 018 or 030. To confirm an effect of 018 and 030 on $\mathrm{A} \beta \mathrm{PP} / \mathrm{A} \beta$ levels, we treated astrocyteneuronal co-cultures obtained from APP/PS1 mice, that produce abnormally high levels of A $\beta P P$ [15], with these compounds. We confirmed that levels of A $\beta P P$ mRNA and A $\beta$ were also significantly reduced (Fig. 2).

When calcineurin/FOXO3 interactions are interfered in vivo after inflammatory injury, brain levels of different neurotrophic factors greatly increased [2]. As indicated above, we 
consider that this neurotrophic response forms part of the resolution of inflammation. Thus, we tested in cultured astrocytes whether a similar neurotrophic effect is observed when calcineurin/FOXO3 interactions are interfered with decoy compounds. We measured IGFI, IGF-II, and FGF-8 because they were previously found to show highest increases after calcineurin/FOXO3 uncoupling [2]. Since interfering with calcineurin/FOXO3 interactions modulates A $\beta$ PP processing (Fig. 2), we also determined the effect of compounds 018 and 030 on $\mathrm{sA} \beta \mathrm{PPa}$ levels, a neurotrophic derivative of $\mathrm{A} \beta \mathrm{PP}[16]$ that modulates $\mathrm{A} \beta \mathrm{PP}$ processing [17]. As shown in Fig. 3, we found that cultured astrocytes exposed to A $\beta$ showed increased levels of these neurotrophic proteins when either decoy compound was added. Moreover, in astrocytes from APP/PS1 mice, these neurotrophic factors also increased in response to compounds 018 and 030 (Fig. 3C). Accordingly, expression of pro-inflammatory cytokines TNFa and IL-6 in astrocytes, that was increased in response to $\mathrm{A} \beta$ challenge, was also attenuated. The inhibitory action of these compounds was seen both in wild type astrocytes exposed to A, and in astrocytes from APP/PS1 mice (Fig. 4).

\section{DISCUSSION}

The present results indicate that interfering calcineurin/FOXO interactions, that take place during pro-inflammatory activation in astrocytes, using decoy molecules, may represent a feasible new approach to treat neuro-inflammatory damage associated to brain amyloidosis in AD. An advantage of this approach is that association of calcineurin with FOXO3 in response to inflammatory stimuli does not take place in neurons, which reduces possible off-target effects. Our observations lend support to future studies using either intranasal delivery of this type of decoy compounds or, alternatively, develop similar compounds able to cross the blood-brain barrier for systemic administration. Because previous in vivo models showed that inhibition of calcineurin/FOXO interactions is sufficient to significantly delay brain amyloidosis, and prevent, or even revert, cognitive decline in AD mice [2], we anticipate similar therapeutic actions for these type of decoy compounds when used in vivo. Furthermore, the present results indicate that the interaction between calcineurin and FOXO3 in astrocytes is also instrumental in neuronal death elicited by $\mathrm{A} \beta$, which opens the possibility that the use of similar compounds in $\mathrm{AD}$ patients may also halt neuronal death. However, this possibility will require additional studies.

Until now, anti-inflammatory approaches to treat AD have failed [18]. This strategy was initiated as a result of epidemiological observations supporting a role of inflammation in $\mathrm{AD}$, as patients receiving non-steroidal anti-inflammatory compounds showed protection against the disease [19]. Diverse short-comings may account for a lack of effect of antiinflammatory therapies in AD patients. Thus, depending on the anti-inflammatory used, 
very different outcomes on inflammatory pathways may be expected. Further, regulation of inflammatory cytokines is context- and stage-dependent, so established tauopathy or functional disturbances related to neuronal loss may not be counterbalanced [20]. However, the strategy that we propose to use is based on cell-specific (astrocytes) pro/antiinflammatory pathways, which until now has not been considered. Activation of calcineurin is important in the response to pro-inflammatory stimuli such as $\mathrm{TNF}$ [21], and we previously documented that calcineurin activation participates in both progression and resolution of inflammation, depending on upstream activators such as TNFa or IGF-I, respectively [1]. By inhibiting the interaction of calcineurin with FOXO3, this pathway is re-directed towards resolution of inflammation. Lowering inflammation will reduce $\mathrm{A} \beta \mathrm{PP}$ synthesis $[22,23]$, as we now observed. In turn, an increased output of neuroprotective mediators will probably reduce amyloidogenic A $\beta P P$ processing $[2,22]$. Indeed, increased levels of $\mathrm{sA} \beta P P a$ together with decreased production of $A \beta$ peptides were detected. sA $\beta P P a$ also inhibits BACE-1 activity, which in turns will reduce $A \beta$ levels [24], as we observed now in cultured astrocytes.

In summary, pharmacological manipulation of an astrocyte-specific pro-inflammatory pathway that interferes with the association of the phosphatase calcineurin with the transcription factor FOXO3, protects neurons against A-induced cell death. Cell-specific anti-inflammatory drugs may open a new avenue to treat neuro-inflammation associated to $\mathrm{AD}$ and other neurodegenerative diseases.

\section{ACKNOWLEDGMENTS}

This work was supported by Ciberned Collaborative Project 2013/01. Calcineurin mutants were a kind gift of JM Redondo (CNIC, Spain). 


\section{REFERENCES}

[1] Fernandez AM, Fernandez S, Carrero P, Garcia-Garcia M, Torres-Aleman I (2007) Calcineurin in reactive astrocytes plays a key role in the interplay between proinflammatory and anti-inflammatory signals. J Neurosci 27, 8745-8756.

[2] Fernandez AM, Jimenez S, Mecha M, Davila D, Guaza C, Vitorica J, TorresAleman I (2012) Regulation of the phosphatase calcineurin by insulin-like growth factor I unveils a key role of astrocytes in Alzheimer's pathology. Mol Psychiatry 17, 705-718.

[3] Abdul HM, Sama MA, Furman JL, Mathis DM, Beckett TL, Weidner AM, Patel ES, Baig I, Murphy MP, LeVine H, Kraner SD, Norris CM (2009) Cognitive decline in Alzheimer's disease is associated with selective changes in calcineurin/NFAT signaling. J Neurosci 29, 12957-12969.

[4] Manolopoulos KN, Klotz LO, Korsten P, Bornstein SR, Barthel A (2010) Linking Alzheimer's disease to insulin resistance: The FoxO response to oxidative stress. Mol Psychiatry 15, 1046-1052.

[5] Miroux B, Walker JE (1996) Over-production of proteins in Escherichia coli: Mutant hosts that allow synthesis of some membrane proteins and globular proteins at high levels. J Mol Biol 260, 289-298.

[6] Sambrook J, Gething MJ (1989) Protein structure. Chaperones, paperones. Nature $342,224-225$.

[7] Grigoriu S, Bond R, Cossio P, Chen JA, Ly N, Hummer G, Page R, Cyert MS, Peti W (2013) The molecular mechanism of substrate engagement and immunosuppressant inhibition of calcineurin. PLoS Biol 11, e1001492.

[8] Molecular Operating Environment (MOE) Chemical Computing Group Inc. 1010 Sherbooke St. West Suite \#910 Montreal QC Canada H3A 2R7. 2013.

[9] Friesner RA, Banks JL, Murphy RB, Halgren TA, Klicic JJ, Mainz DT, Repasky MP, Knoll EH, Shelley M, Perry JK, Shaw DE, Francis P, Shenkin PS (2004) Glide: A new approach for rapid, accurate docking and scoring. 1. Method and assessment of docking accuracy. J Med Chem 47, 1739-1749.

[10] Duan J, Dixon SL, Lowrie JF, Sherman W (2010) Analysis and comparison of 2D fingerprints: Insights into database screening performance using eight fingerprint methods. J Mol Graph Model 29, 157-170. 
[11] Trejo JL, Carro E, Torres-Aleman I (2001) Circulating insulin-like growth factor I mediates exercise-induced increases in the number of new neurons in the adult hippocampus. J Neurosci 21, 1628-1634.

[12] Fernandez AM, Gonzalez de la Vega A, Torres-Aleman I (1998) Insulin-like growth factor I restores motor coordination in a rat model of cerebellar ataxia. Proc Natl Acad Sci USA 95, 1253-1258.

[13] Garwood CJ, Pooler AM, Atherton J, Hanger DP, Noble W (2011) Astrocytes are important mediators of Abeta-induced neurotoxicity and tau phosphorylation in primary culture. Cell Death Dis 2, e167.

[14] Wang C, Wurtman RJ, Lee RK (2000) Amyloid precursor protein and membrane phospholipids in primary cortical eurons increase with development, or after exposure to nerve growth factor or Abeta(1-40). Brain Res 865, 157-167.

[15] Trinchese F, Liu S, Ninan I, Puzzo D, Jacob JP, Arancio O (2004) Cell cultures from animal models of Alzheimer's disease as a tool for faster screening and testing of drug efficacy. J Mol Neurosci 24, 15-21.

[16] Perez RG, Zheng H, Van der Ploeg LH, Koo EH (1997) The beta-amyloid precursor protein of Alzheimer's disease enhances neuron viability and modulates neuronal polarity. J Neurosci 17, 9407-9414.

[17] Obregon D, Hou H, Deng J, Giunta B, Tian J, Darlington D, Shahaduzzaman M, Zhu Y, Mori T, Mattson MP, Tan J (2012) Soluble amyloid precursor protein-alpha modulates beta-secretase activity and amyloid-beta generation. Nat Commun 3, 777.

[18] Jaturapatporn D, Isaac MG, McCleery J, Tabet N (2012) Aspirin, steroidal and non-steroidal anti-inflammatory drugs for the treatment of Alzheimer's disease. Cochrane Database Syst Rev 2, CD006378.

[19] McGeer PL, Schulzer M, McGeer EG (1996) Arthritis and anti-inflammatory agents as possible protective factors for Alzheimer's disease: A review of 17 epidemiologic studies. Neurology 47, 425-432.

[20] Heneka MT, Carson MJ, Khoury JE, Landreth GE, Brosseron F, Feinstein DL, Jacobs AH, Wyss-Coray T, Vitorica J, Ransohoff RM, Herrup K, Frautschy SA, Finsen B, Brown GC, Verkhratsky A, Yamanaka K, Koistinaho J, Latz E, Halle A, Petzold GC, Town T, Morgan D, Shinohara ML, Perry VH, Holmes C, Bazan NG, Brooks DJ, Hunot S, Joseph B, Deigendesch N, Garaschuk O, Boddeke E, Dinarello CA, Breitner JC, Cole GM, 
Golenbock DT, Kummer MP (2015) Neuroinflammation in Alzheimer's disease. Lancet Neurol 14, 388-405.

[21] Goldfeld AE, Tsai E, Kincaid R, Belshaw PJ, Schrieber SL, Strominger JL, Rao A (1994) Calcineurin mediates human tumor necrosis factor alpha gene induction in stimulated T and B cells. J Exp Med 180, 763-768.

[22] Brugg B, Dubreuil YL, Huber G, Wollman EE, Delhaye-Bouchaud N, Mariani J (1995) Inflammatory processes induce beta-amyloid precursor protein changes in mouse brain. Proc Natl Acad SciUSA 92, 3032-3035.

[23] Forloni G, Demicheli F, Giorgi S, Bendotti C, Angeretti N (1992) Expression of amyloid precursor protein mRNAs in endothelial, neuronal and glial cells: Modulation by interleukin-1. Brain Res Mol Brain Res 16, 128-134.

[24] Peters-Libeu C, Campagna J, Mitsumori M, Poksay KS, Spilman P, Sabogal A, Bredesen DE, JohnV (2015) sAbetaPPalpha is a potent endogenous inhibitor of BACE1. J Alzheimers Dis 47, 545-555. 


\section{CAPTIONS TO FIGURES}

Figure 1. Decoy compounds that interfere with calcineurin and FOXO3 interactions. A) 3D model of the complex of calcineurin with the calcineurin-inhibiting domain of the African swine fever virus protein A238L. The position of the substrate recognition site $\varphi \mathrm{LxVP}$ is shown. Calcineurin A (CNA) and B (CNB) moieties are indicated. B) Example of the screening system used to identify compounds that interfere with the association of calcineurin with FOXO3 upon stimulation of astrocytes with LPS (10 nM). ACN compounds 018 and 030 displayed the highest activity. Lanes: 1: vehicle (DMSO), 2: Dexamethasone+LPS, 3: vehicle+LPS, 4: ACN015+LPS, 5: ACN016+LPS, 6: ACN017+LPS, 7: ACN018+LPS, 8: Vehicle, 9: Dexamethasone+LPS, 10: Vehicle+LPS, 11: ACN026+LPS, 12: ACN029+LPS, 13: ACN030+LPS. All compounds were tested at $10 \mu \mathrm{M} . \mathrm{C})$ Neurons, but not astrocytes, die after challenged with $\mathrm{A} \beta(2 \mu \mathrm{M})$. Thus, activated caspase 3 staining (red) was found only in neuronal cultures ( $\beta 3$-tubulin + cells in green) but not in astrocytes (GFAP+, in green). D) Neurons (Neu) in culture do not show association of calcineurin with FOXO3 after LPS or A $\beta$ challenge. Cultured astrocytes (As) that show association of FOXO3 with calcineurin were used as positive controls. E) Compounds ACN 018 and 030 showed the greatest inhibitory activity on A $\beta$-induced neuronal death. Neurons were cultured with astrocytes and challenged with $A \beta(2 \mu \mathrm{M})$. Twenty-four hours later activated caspase levels were measured by western blot in culture lysates. Bars in the right show quantification of blots. Lanes: 1: Control, 2,3: $A \beta, 4,5$ : ACN018+A $\beta, 6,7:$ ACN030+A, 8: ACN030. $(* * \mathrm{p}<0.01$ versus $\mathrm{A} \beta$ and $* * * \mathrm{p}<0.001$ versus control, $\mathrm{n}=3$ ). All compounds were tested at $10 \mu \mathrm{M}$. When given alone, neither compound affected neuronal viability (not shown).

Figure 2. Interfering calcineurin/FOXO3 interactions reduces levels of $A \beta P P$ mRNA and its amyloidogenic processing. A) ACN018 and 030 reduce levels of A PPP mRNA in wild type astrocyte-neuronal co-cultures exposed to A, and in astrocyte-neuronal co-cultures from APP/PS1 mice, B) Similarly, ACN018 and 030 reduce levels of A $\beta$ in wild type astrocyte-neuronal co-cultures exposed to $A \beta$ and in astrocyte-neuronal co-cultures from APP/PS1 mice. Representative blots using in combination anti-A $\beta$ antibodies $6 \mathrm{E} 10$ and 82E1. Monomer and dimer forms of $A \beta$ are shown. Quantitation histograms are shown below. $\left({ }^{*} \mathrm{p}<0.05,{ }^{* *} \mathrm{p}<0.01\right.$ and ${ }^{* * *} \mathrm{p}<0.001$ versus control, $\mathrm{n}=5$ ).

Figure 3. ACN018 and 030 stimulate the release of neurotrophic factors in astrocytes exposed to inflammatory challenge. A, B) In the presence of LPS (A) or A $\beta$ (B), ACN018 and 030 stimulate the secretion of IGF-I, IGF-II, FGF-8, and sA $\beta P P a$. C) Astrocytes derived from APP/PS1 mice show enhanced secretion of IGF-I, IGF-II, FGF-8, and 
sA $\beta P P a$ when cultured in the presence of ACN018 or 030 . Normalization of protein load was performed with bovine serum albumin (BSA) from culture supernatants.

Figure 4. Compounds 018 and 030 decrease mRNA levels of pro-inflammatory cytokines TNFa (left chart) and IL-6 (right) that are increased after exposure of wild type astrocytes to A $\beta$ or in APP/PS1 astrocytes $(* \mathrm{p}<0.05, * * \mathrm{p}<0.01$ and $* * * \mathrm{p}<0.001$ versus control, $\mathrm{n}=$ $5)$. 
FIGURE 1

a)

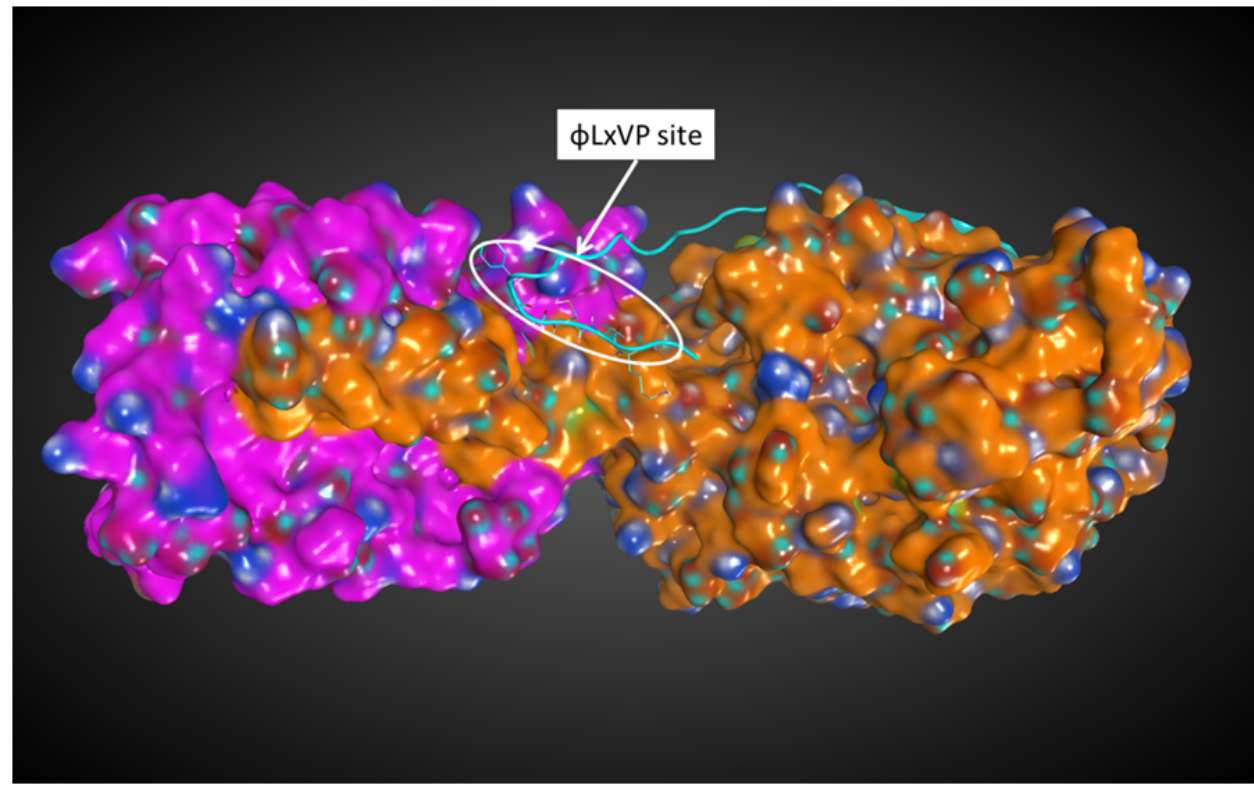

b)

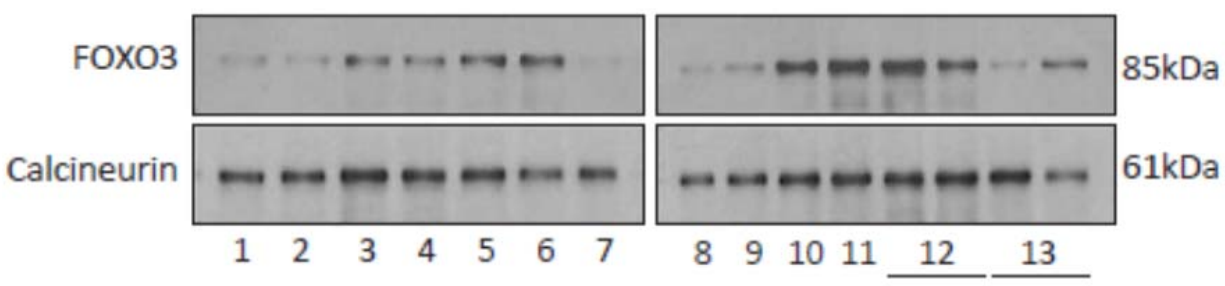

c)
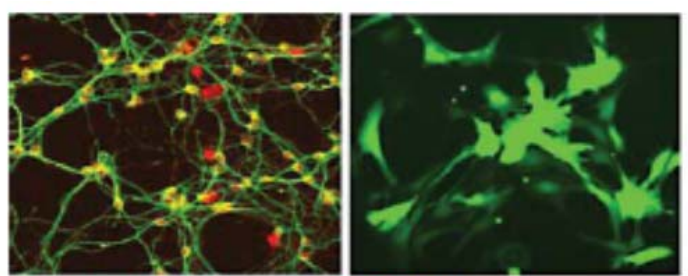

$B_{3}$ Tubulin/cleaved caspase 3 GFAP/cleaved caspase 3 
d)

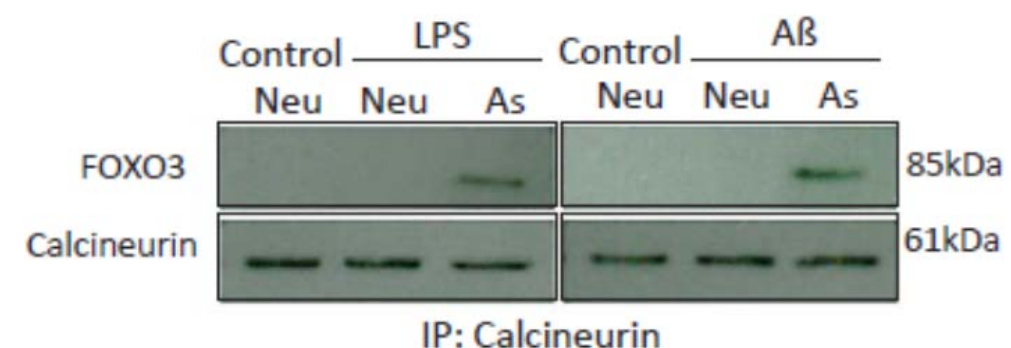

e)

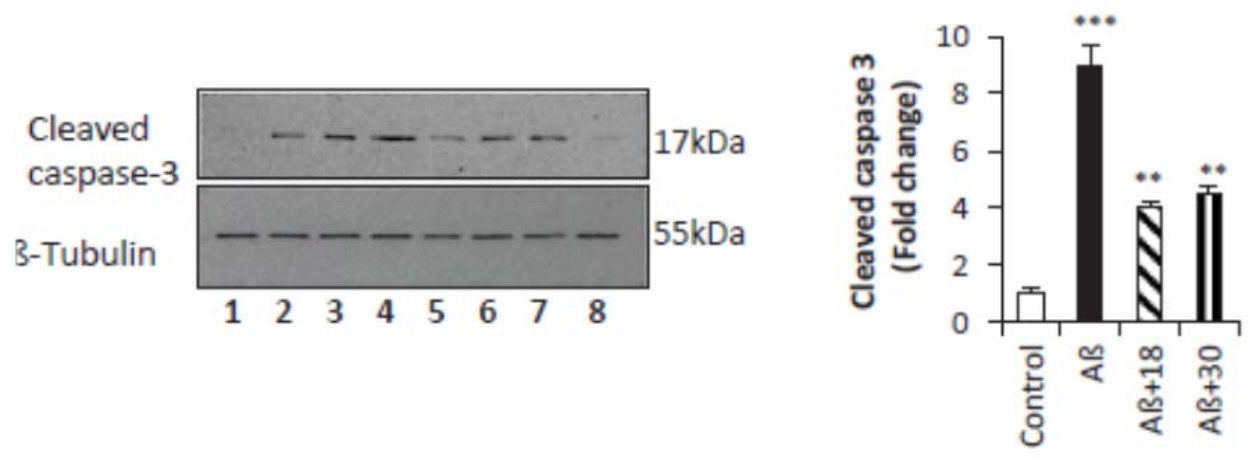


FIGURE 2

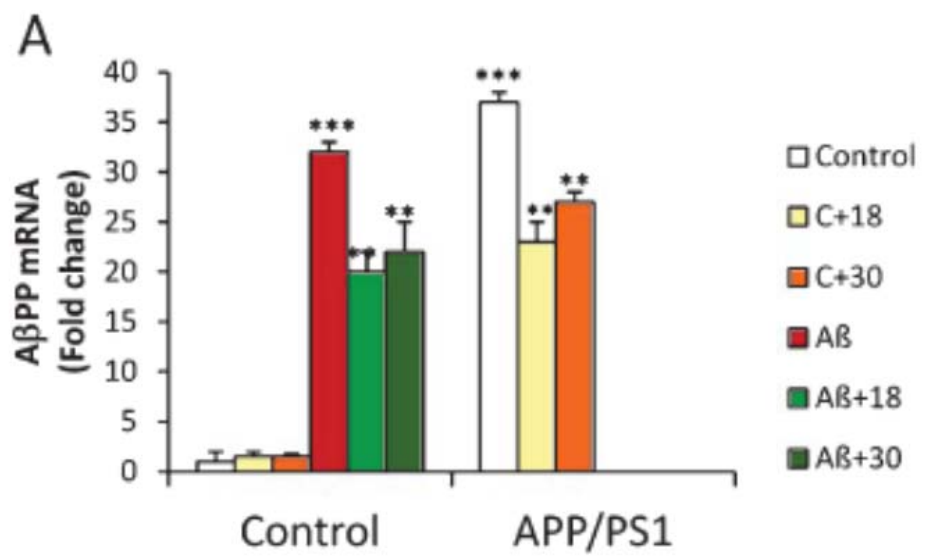

B
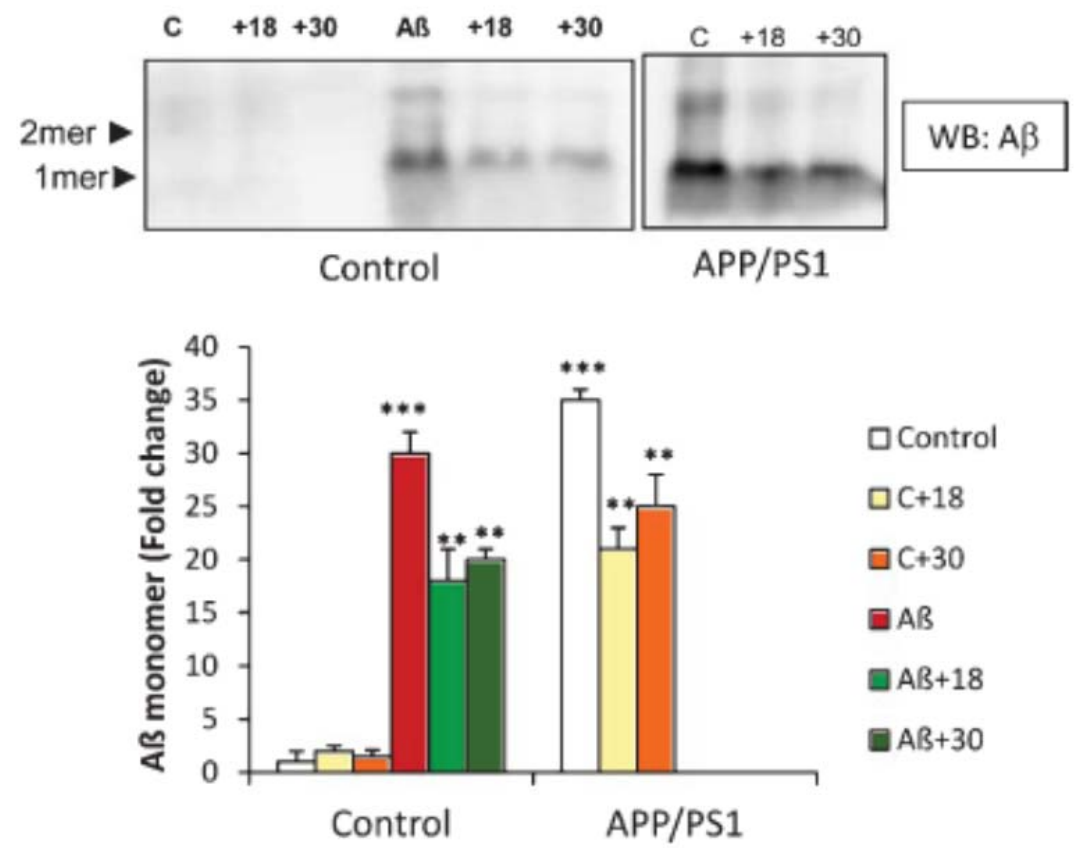
FIGURE 3

A

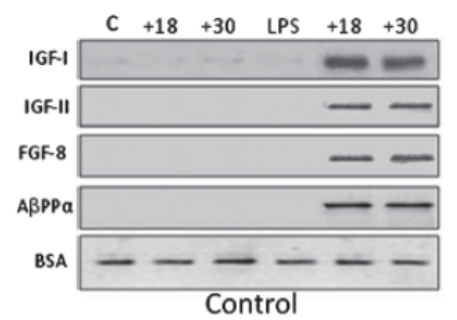

B

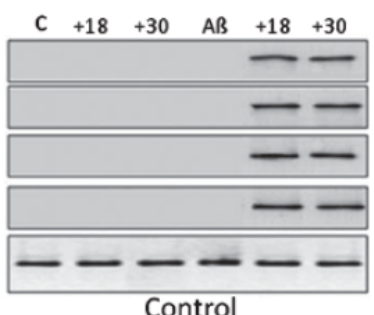

C

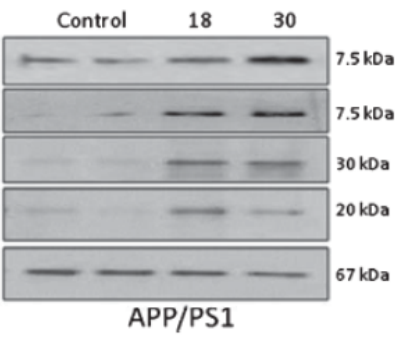


FIGURE 4
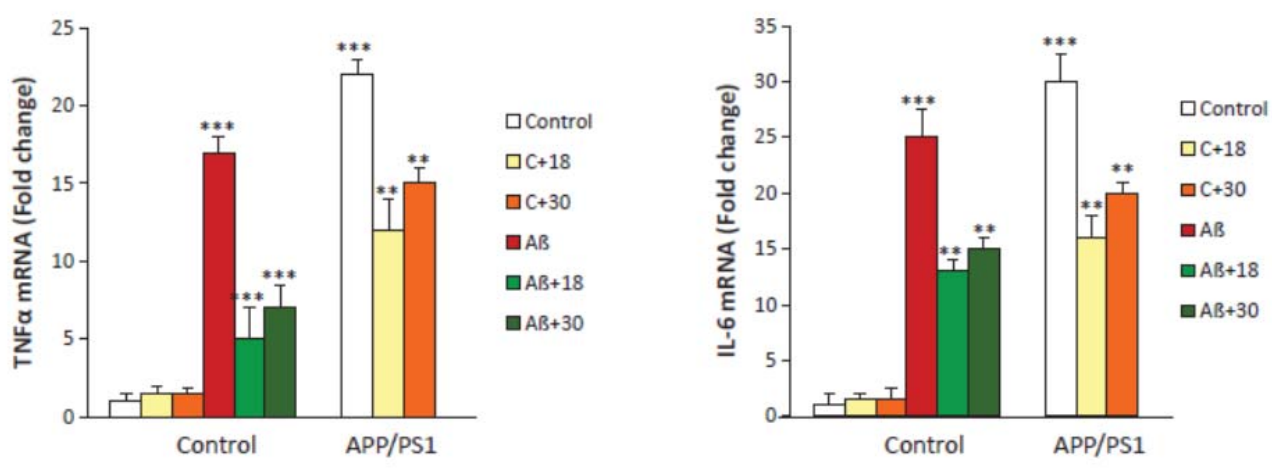